\title{
) Screening of antioxidant and antimicrobial activities on Plectranthus spp. extracts
}

\author{
Pesquisa das actividades antioxidante e antimicrobiana em extractos de plantas do \\ género Plectranthus
}

\author{
Patrícia Rijo ${ }^{1,2,3^{*}}$, Marina Batista ${ }^{1,2}$, Marisa Matos $^{2}$, Helga Rocha ${ }^{2}$, Sandra Jesus ${ }^{2}$, M. Fátima Simões ${ }^{3}$ \\ ${ }^{1}$ CBIOS - Universidade Lusófona's Research Center for Health Science and Technologies, Laboratory of \\ Pharmacology and Therapeutics - Campo Grande, 376, 1749-024, Lisboa, Portugal \\ ${ }^{2}$ ERISA, Escola Superior de Saúde de Ribeiro Sanches, Rua do Telhal aos Olivais, n. ${ }^{\circ} 8$ - $8^{\mathrm{a}}$, \\ 1900-693 Lisboa - Portugal \\ ${ }^{3}$ Research Institute for Medicines and Pharmaceutical Sciences (iMed.UL), Faculdade de Farmácia, \\ Universidade de Lisboa, Av. Prof. Gama Pinto, 1649-003 Lisboa, Portugal \\ Email: patricia.rijo@ulusofona.pt
}

\begin{abstract}
The use of medicinal plants is a widespread tendency in folk medicine. The Lamiaceae family contains several genera such as sage (Salvia), basil (Ocimum) and mint (Mentha) with a rich diversity of ethnobotanical uses. This study presents the in vitro antibacterial and antioxidant activities evaluation of five species extracts of the Plectranthus genus: $\mathrm{P}$. hadiensis (vr), P. madagascarensis, P. neochilus, P. verticillatus e P. barbatus. Aqueous and acetonic extracts were obtained by five different methods: infusion, decoction, ultrasounds, microwave and maceration $(10 \% \mathrm{~W} / \mathrm{v}$ of dry plant $)$. The highest amount of dry extract was obtained by the infusion method for Plectranthus barbatus $(22,0 \pm 1,0$ to $33,0 \pm 3,0$ $\mathrm{mg} / \mathrm{mL}$ ). The extracts obtained were tested for their antimicrobial activity against three Gram-positive bacteria and two Gram-negative bacteria using the well diffusion method. Only the acetonic extracts of P. madagascarensis, P. hadiensis (vd) and P. verticillatus showed antibacterial activity and this was exclusively against Gram-positive bacteria. The highest antimicrobial activity was exhibited by P. madagascarensis extract with inhibition zones ranging from 23 to $36 \mathrm{~mm}$. The antioxidant activity of the extracts was qualitatively determined using the 2,2-diphenyl-1-picrylhydrazyl (DPPH) radical assay in tlc and yielded positive results for the majority of the extracts studied.
\end{abstract}

Keywords: Plectranthus, antioxidant activity, antimicrobial activity.

\begin{abstract}
Resumo
O uso de plantas medicinais é uma tendência generalizada na medicina popular. O género Plectranthus pertence à família das Lamiaceae tal como a salva (Salvia), o manjericão (Ocimum) e a hortelã (Mentha), que apresentam uma grande diversidade de usos etnobotânicos. No presente trabalho, foram avaliadas as atividades antimicrobiana e antioxidante de extractos de cinco espécies do género Plectranthus: P. hadiensis (vd), P. madagascarensis, P. neochilus, P. verticillatus e P. barbatus. Os extractos foram obtidos por diversos métodos: infusão, decocção, ultra-sons, micro-ondas e maceração (10\% $\mathrm{m} / \mathrm{v}$ de planta seca) com dois solventes diferentes (água e acetona). O método de infusão permitiu obter a maior quantidade de extrato seco a partir da planta Plectranthus barbatus $(22 \pm 1,0-33 \pm 3,0 \mathrm{mg} / \mathrm{mL})$. A actividade antimicrobiana dos extratos obtidos foi determinada usando o método da difusão em poço e foi testada contra três bactérias Gram-positivas e duas bactérias Gram-negativas. Só os extratos acetónicos de P. madagascarensis, P. verticillatus e P. hadiensis (vd) demonstraram ser ativos e exclusivamente em bactérias Gram-positivas. O extrato de P. madagascarensis demonstrou a maior actividade antibacteriana com halos de inibição entre 23 e $36 \mathrm{~mm}$. A atividade antioxidante dos extratos foi determinada qualitativamente usando o método do radical 2,2-difenil-1-picril-hidrazina (DPPH) em ccd obtiveram-se resultados positivos na maioria dos extractos estudados.
\end{abstract}

Palavras-chave: Plectranthus, atividade antioxidante, atividade microbiana. 


\section{Introduction}

The discovery and development of drugs from medicinal plant sources continue to provide new and important leads against various pharmacological targets. Drugs derived from medicinal plants can serve not only as new drugs themselves but also as drug leads suitable for optimization by medicinal and synthetic chemists. Even when new chemical structures are not found during drug discovery from medicinal plants, known compounds with new biological activity can also provide important drug leads. Several known compounds isolated from traditionally used medicinal plants, have already been shown to act on newly validated molecular targets ${ }^{(1)}$.

Medicinal plants, which form the backbone of traditional medicine, have in the last few decades been the subject of very intense pharmacological studies. In developing countries, it is estimated that about $80 \%$ of the population rely on traditional medicine for their primary health care. Therefore, there is a need to screen medicinal plants for potential bioactive compounds of therapeutic value and as sources of lead compounds in drug development ${ }^{(2,3)}$.

The Plectranthus genus belongs to the Lamiaceae family and has about 350 species that are distributed in tropical and subtropical regions of Asia, Africa, Australia, Madagascar, India and the Pacific Islands. Some Plectranthus species that are native to Africa were introduced in the New World, following the Portuguese Discoveries in the $16^{\text {th }}$ century. These plants present ornamental, medicinal, economical and a rich diversity of ethnobotanical uses. Several species are used throughout the world as antihelmintics, antiseptics and to treat a wide range of diseases (4). Phytochemical studies using some species of Plectranthus revealed the presence of a large number of diterpenes and triterpenes. The terpenoids are considered to be the main responsible compounds for the antimicrobial and antioxidant activities presented by Plectranthus species ${ }^{(3)}$.

Antioxidants mitigate the harmful effect of free radicals, which damage human cells under 'oxidative stress' conditions. Moreover, an imbalance of free radicals may cause serious disturbances in cell metabolism. Free radicals are unstable species because they have unpaired electrons and seek stability through electron pairing with biological macromolecules ${ }^{(5)}$. Active oxygen (hydroxyl, peroxyl radicals and singlet oxygen) is highly toxic and an important causative agent of many diseases including cancer, heart disease, cataract and congestive disorders. Antioxidant compounds block the oxidation processes that produce free radicals which contribute to these chronic diseases and ageing (6). Recently, there has been increased concern about synthetic antioxidants, partly due to their possible toxicity against animal DNA. This may explain both the interest in examining plant extracts as a source of cheaper and effective antioxidants and the growing interest in nutraceuticals ${ }^{(5)}$. It is recognized that 226

\section{Introdução}

A descoberta de fármacos a partir de plantas medicinais continua a fornecer novas e importantes pistas contra diversos alvos farmacológicos. Os fármacos derivados de plantas medicinais podem servir não apenas como novos fármacos em si, mas também como fármacos protótipo adequados para otimização por químicos medicinais e de síntese. Mesmo quando não são encontradas novas estruturas químicas durante a descoberta de fármacos a partir de plantas medicinais, os compostos conhecidos com novas atividades biológicas podem fornecer importantes protótipos de fármacos. Vários compostos conhecidos, isolados a partir de plantas medicinais com uso tradicional, já demostraram ser ativos em novos alvos moleculares validados ${ }^{(1)}$.

Nas últimas décadas, as plantas medicinais, que constituem a base da medicina tradicional, têm sido sujeitas a estudos farmacológicos muito intensos. Nos países em desenvolvimento estima-se que cerca de $80 \%$ da população dependa da medicina tradicional para os cuidados primários de saúde. Sendo assim, surge a necessidade de fazer uma triagem das plantas medicinais para obtenção de potenciais compostos bioativos de valor terapêutico e como fontes de compostos protótipo no desenvolvimento de fármacos ${ }^{(2,3)}$.

O género Plectranthus pertencente à família Lamiaceae tem cerca de 350 espécies que são distribuídas em regiões tropicais e subtropicais da Ásia, África, Austrália, Madagáscar, Índia e Ilhas do Pacífico. Algumas espécies de Plectranthus, que são nativas de África foram introduzidas no Novo Mundo, seguindo os Descobrimentos Portugueses (século XVI). Estas plantas têm interesse ornamental, medicinal, económico e uma rica diversidade de usos etnobotânicos. Várias espécies são utilizadas em todo o mundo como antihelmínticos, anti-sépticos e de purga contra uma ampla gama de doenças ${ }^{(4)}$. Estudos fitoquímicos que utilizam algumas espécies de Plectranthus revelaram a presença de um grande número de diterpenos e triterpenos. Os terpenóides são considerados os principais compostos responsáveis pelas actividades antimicrobiana e antioxidante presentes nas espécies de Plectranthus ${ }^{(3)}$.

Os antioxidantes atenuam os efeitos nocivos dos radicais livres, que danificam as células humanas sob condições de "stress oxidativo". Um desequilíbrio de radicais livres pode ainda causar perturbações graves no metabolismo celular. Os radicais livres são espécies instáveis porque têm eletrões desemparelhados que procuram a estabilidade através do emparelhamento de eletrões com macromoléculas biológicas ${ }^{(5)}$. O oxigénio ativo (hidroxilo, radicais peroxilo e oxigénio singuleto) é altamente tóxico e um importante agente que causa muitas doenças, incluindo cancro, doenças cardíacas, cataratas e insuficiência cardíaca. Os compostos antioxidantes bloqueiam os processos de oxidação que produzem radicais livres que contribuem para estas doenças crónicas e envelhecimento ${ }^{(6)}$. Recentemente, 
antioxidant (mainly polyphenolic) compounds from plant extracts can act by free radical scavenging, singlet oxygen quenching and chelating of transitional metal such as iron. These compounds can also act as reducing agents and activators of antioxidative defense enzyme systems to suppress radical damage in the biological system ${ }^{(7)}$. In recent works, the antioxidant activity of Plectranthus sp., the aqueous extract of P. barbatus leaves ${ }^{(8)}$ and the ethanol extract of P. amboinicus ${ }^{(9)}$ was studied. Regarding the antioxidant properties of the Lamiaceae family and future phytochemical evaluation of active compounds, there are several related reports of Plectranthus spp. plants extracts in the literature ${ }^{(3)}$.

Natural products have been a rich source in supplying products for the development of drugs for the treatment of bacterial infections. The pressure to find new antibiotics with new modes of action has led to the investigation of plant sources such as antimicrobials. Since plants produce natural products as chemical defenses against microorganisms in their environment, research into plants is a logical choice due to this ecological rationality ${ }^{(10)}$. Many plants have been studied scientifically in order to confirm their antimicrobial action. This is done through the study of plant extracts. Some Plectranthus spp. with ethnopharmacological applications have been used for their antibiotic action. Many biological activities have been attributed to this genus and the antimicrobial activity is often cited ${ }^{(11)}$. Indeed, it is known that both extracts and metabolites of several Plectranthus spp. often show antimicrobial activities and the antimicrobial capability of some of them was evaluated against Gram-positive and Gramnegative bacteria and the yeast Candida albicans ${ }^{(12)}$.

The aim of this work was to prepare and evaluate the antimicrobial and antioxidant activities of different extracts from five Plectranthus plants: $P$. hadiensis, $P$. madagascarensis, $P$. neochilus, $P$. verticillatus and $P$. barbatus. In this study, extracts were prepared by five different methods (infusion, decoction, ultrasonic, microwave and maceration) using water or acetone as extraction solvents. Their antibacterial and antioxidant activities were determined by means of the well diffusion assay and the DPPH radical qualitative method, respectively. tem havido uma preocupação crescente sobre antioxidantes sintéticos, em parte devido à sua possível toxicidade contra DNA animal. Isto pode explicar o interesse em examinar extratos de plantas como uma fonte de antioxidantes mais barata e mais eficaz e o crescente interesse em produtos nutracêuticos ${ }^{(5)}$. É reconhecido que os compostos antioxidantes (principalmente compostos polifenólicos) obtidos a partir de extratos de plantas podem actuar por sequestro de radicais livres, captura de oxigénio singuleto, formação de quelatos com metais de transição como o ferro, assim como agentes redutores e activadores de sistemas de enzimas antioxidantes de defesa para suprimir os danos dos radicais no sistema biológico ${ }^{(7)}$. Em trabalhos recentes, a atividade antioxidante de Plectranthus sp., do extrato aquoso de folhas de $P$. barbatus ${ }^{(8)}$ e do extrato etanólico de P. amboinicus ${ }^{(9)}$ foi estudada. Considerando as propriedades antioxidantes descritos na família Lamiaceae, e a futura avaliação fitoquímica de compostos ativos, existem vários estudos relacionados com extratos de plantas Plectranthus spp. na literatura ${ }^{(3)}$.

Os produtos naturais têm sido uma fonte rica em fornecer pistas para o desenvolvimento de fármacos para o tratamento de infeções bacterianas. A pressão para encontrar novos antibacterianos com novos modos de ação tem conduzido à exploração de fontes vegetais como antimicrobianos. A escolha é lógica dada a racionalidade ecológica que as plantas produzem produtos naturais como uma defesa química contra microorganismos do seu ambiente ${ }^{(10)}$. Muitas plantas têm sido estudados cientificamente, a fim de confirmar a sua ação antimicrobiana através do estudo dos seus extratos. Algumas espécies de Plectranthus com aplicações etnofarmacológicas são usados pela sua ação antibiótica. Muitas atividades biológicas têm sido atribuídas a este género e a atividade antimicrobiana é muito citada ${ }^{(11)}$. Com efeito, sabe-se que ambos os extractos e os metabolitos de vários Plectranthus spp. apresentam frequentemente actividades antimicrobianas e o potencial antimicrobiano de alguns deles foi avaliado contra bactérias Gram-positivas e Gram-negativas e a levedura Candida albicans ${ }^{(12)}$

O objetivo deste trabalho foi o de preparar e investigar as atividades antimicrobiana e antioxidante de diferentes extratos de cinco plantas: $P$. hadiensis, $P$. madagascarensis, $P$. neochilus, $P$. verticillatus e $P$. barbatus. Neste estudo, os extractos foram preparados por cinco métodos diferentes (infusão, decocção, microondas, ultra-sons e maceração), utilizando como solventes de extracção água ou acetona. As actividades antibacteriana e antioxidante foram determinadas através do ensaio de difusão em poços e o método qualitativo do radical DPPH, respectivamente. 


\section{Material and Methods}

\section{Chemicals}

Dimethyl sulphoxide (DMSO) 99.9\% and methanol p.a. were purchased from Merck; 2,2-diphenyl-1picrylhydrazyl (DPPH) was obtained from SigmaAldrich.

\section{Plantmaterials}

Plectranthus hadiensis (vd), Plectranthus madagascarensis, Plectranthus neochilus, Plectranthus barbatus and Plectranthus verticillatus were provided by the Faculty of Pharmacy, University of Lisbon.

\section{Extraction methodologies of plant material}

Aqueous plant extracts were prepared as decoctions, infusions or using microwave or ultrasounds. Acetone extracts were prepared by maceration and ultrasounds. In all procedures dried and powdered plant material (10 g) was extracted using $100 \mathrm{~mL}$ of solvent (water or acetone) and then filtered through Whatman paper by:

Infusion

Extracts were prepared using the plant material in freshly boiled distilled water for $10 \mathrm{~min}$.

\section{Decoction}

Extracts were prepared using the plant material boiled in the distilled water for $10 \mathrm{~min}$.

\section{Microwave}

Extracts were prepared using the plant material and distilled water in a conventional microwave for 2 minutes at a power of $800 \mathrm{~W}$.

\section{Maceration}

Plant material and acetone were left stirring at room temperature. After 24 hours the macerate was filtered into a round bottom flask and then evaporated (under vacuum, 40 ${ }^{\circ} \mathrm{C}$ ) yielding a residue.

\section{Ultrasound}

Plant material and acetone were placed in the ultrasonic bath at room temperature for 1 hour. The acetone extract was filtered into a round bottom flask and then evaporated

\section{Materiais e Métodos}

\section{Reagentes}

Dimetilsulfóxido (DMSO) 99,9\% e metanol p.a. foram adquiridos à Merck; 2,2-difenil-1-picril-hidrazilo (DPPH) foi obtido da Sigma-Aldrich.

\section{Material vegetal}

Plectranthus hadiensis (variedade tomentosus), Plectranthus madagascarensis, Plectranthus neochilus, Plectranthus verticillatus e Plectranthus barbatus foram fornecidos pela Faculdade de Farmácia da Universidade de Lisboa.

\section{Metodologias de extracção do material vegetal}

Os extratos vegetais aquosos foram preparados como infusões, decocções, utilizando microondas ou ultrasons. Os extratos de acetona foram preparados por maceração e ultra-sons. Em todos os processos, o material vegetal seco e em pó $(10 \mathrm{~g})$ foi extraído com 100 $\mathrm{mL}$ de solvente (água ou acetona) e, em seguida, filtrado através de papel Whatman por:

Infusões

Os extractos foram preparados usando o material vegetal em água destilada recentemente fervida durante 10 minutos.

Decocção

Os extractos foram preparados utilizando os materiais vegetais fervidos em água destilada durante $10 \mathrm{~min}$.

\section{Microondas}

Os extractos foram preparados usando o material de planta e a água destilada num microondas convencional, durante 2 minutos a uma potência de $800 \mathrm{~W}$.

\section{Maceração}

O material das plantas e a acetona foram deixados sob agitação à temperatura ambiente. Após 24 horas, o macerado foi filtrado para um balão de fundo redondo e, em seguida, a evaporação (em vácuo, $40^{\circ} \mathrm{C}$ ) originou um resíduo.

\section{Ultra-sons}

O material das plantas e a acetona foram colocados num banho de ultra-sons à temperatura ambiente durante 1 hora. $\mathrm{O}$ extracto de acetona foi filtrado para um balão de fundo 
until dry (under vacuum, $40^{\circ} \mathrm{C}$ ) yielding a residue.

Lyophilization of aqueous extracts

For all extraction methods (infusion, decoction, ultrasounds and microwave) aliquots of $1 \mathrm{ml}$ of each extract were lyophilized and used to determine the dry weight. Data presented in Table 1 are the average of three independent measures.

\section{Microorganisms}

The microorganisms used in this study were obtained from the American Type Culture Collection (ATCC). They included five bacterial species (Staphylococcus aureus ATCC 25923, Bacillus subtilis ATCC 6633, Enterococcus faecalis ATCC 29212, Escherichia coli ATCC 25922 and Pseudomonas aeruginosa ATCC 27853). Microorganisms were grown at $37^{\circ} \mathrm{C}$ in MullerHinton culture media.

\section{Antimicrobial activity}

The well diffusion assay was used to determine the antimicrobial activity of the extracts ${ }^{(13)}$. Petri dishes containing $20 \mathrm{ml}$ Mueller-Hinton culture medium were inoculated with $0.1 \mathrm{ml}$ of a bacterial cell suspension matching a $0.5 \mathrm{McF}$ arland standard solution. The suspension was uniformly spread using a sterile swab over the surface of the medium. Wells of approximately $5 \mathrm{~mm}$ in diameter were made in the agar plates with a sterile glass Pasteur pipette and $50 \mu \mathrm{L}$ of each extract $(1 \mathrm{mg} / \mathrm{ml})$, previously reconstituted by dissolving in dimethyl sulfoxide (DMSO), was added into the well. Sterile DMSO was used as a negative control, while vancomycin $(1 \mathrm{mg} / \mathrm{ml})$ and norfloxacin $(1 \mathrm{mg} / \mathrm{ml})$ were used as positive controls for Grampositive and Gram-negative bacteria, respectively. The plates were then incubated at $37^{\circ} \mathrm{C}$ for 24 hours. The antibacterial activity was assayed by measuring the diameter of the inhibition zone formed around the wells. Each assay was performed in duplicate.

\section{Antioxidant activity}

Antioxidant activity - DPPH free radical-scavenging assay

To obtain a preliminary evaluation of the antioxidant capacity, a DPPH bleaching assay was used as a rapid tlc screening method ${ }^{(14)}$.

\section{TLC-DPPHbleaching assay}

A tlc was performed by applying $10 \mu \mathrm{L}$ of each of the extracts $(1 \mathrm{mg} / \mathrm{mL})$ to silica gel GF254 using the redondo e, em seguida, a evaporação até à secura (sob vácuo, $40^{\circ} \mathrm{C}$ ) originou um resíduo.

\section{Liofilização de extratos aquosos}

Para todos os métodos de extracção (infusão, decocção, ultra-sons e de microondas) alíquotas de $1 \mathrm{~mL}$ de cada extracto foram liofilizados e foram usados para determinar o peso seco. Os dados apresentados na Tabela 1 são a média de três medidas independentes.

\section{Microrganismos}

Os microrganismos usados neste estudo foram obtidos da American Type Culture Collection (ATCC) e incluíram 5 espécies bacterianas (Staphylococcus aureus ATCC 25923, Bacillus subtillis ATCC 6633, Enterococcus faecalis ATCC 29212, Escherichia coli ATCC 25922 e Pseudomonas aeruginosa ATCC27853). Os microrganismos foram cultivados a $37^{\circ} \mathrm{C}$ em meio de cultura Mueller-Hinton.

\section{Atividade antimicrobiana}

Para determinação da actividade antibacteriana dos extractos utilizou-se o método de difusão em poços ${ }^{(13) .}$ Foram preparadas suspensões bacterianas equivalentes a 0.5 na escala de McFarland e $0.1 \mathrm{ml}$ de cada suspensão foi inoculada em placas de petri contendo $20 \mathrm{ml}$ de meio de cultura Mueller-Hinton. A suspensão foi uniformemente distribuída na superfície da placa com uma zaragatoa estéril. Com a ajuda de uma pipeta de Pasteur estéril fizeram-se poços de aproximadamente $5 \mathrm{~mm}$ de diâmetro no agar. A cada poço, foi adicionado $50 \mu \mathrm{L}$ de extracto $(1 \mathrm{mg} / \mathrm{ml})$ previamente reconstituído em dimetilsulfoxido (DMSO). Utilizou-se o DMSO como controlo negativo e a vancomicina ou a norfloxacina com controlos positivos para bactérias Gram-positivas ou bactérias Gram-negativas, respectivamente. As placas foram incubados a $37^{\circ} \mathrm{C}$ durante 24 horas, após as quais se mediu o diâmetro da zona de inibição em redor dos poços. Cada ensaio foi realizado em duplicado.

\section{Atividade antioxidante}

Atividade antioxidante - ensaio da captação do radicallivre DPPH

Para se obter uma avaliação preliminar da capacidade antioxidante, o ensaio de DPPH de descoloração foi usado como um método de rastreio rápido em $\operatorname{ccd}^{(14)}$.

\section{Ensaio de descoloração ccd-DPPH}

Foi realizada uma ccd aplicando $10 \mu \mathrm{L}$ de cada um dos extractos $(1 \mathrm{mg} / \mathrm{mL})$ sobre uma placa de sílica-gel GF254 
corresponding extraction solvent (water or acetone). After drying, the tlc plates were sprayed with a $0.2 \%$ (V/V) DPPH solution in methanol and examined $10 \mathrm{~min}$. after. BHT and quercetin were used as positive controls. Extracts with the capacity to reduce DPPH appeared as yellow spots against a purple background.

\section{Results}

In this work the extracts of the whole plant Plectranthus hadiensis (variety tomentosus), Plectranthus madagascarensis, Plectranthus neochilus, Plectranthus verticillatus and Plectranthus barbatus were prepared by different extraction methods. In order to establish the results as a function of the dry weight of the aqueous extracts, $1 \mathrm{ml}$ of each extract was lyophilized and its dry weight was determined. The results are shown in Table 1. It can be seen that $P$. hadiensis (variety tomentosus) decoction, and P. barbatus - infusion gave the highest yield of plant extract $(32 \pm 2,0$ and $33 \pm 3,0 \mathrm{mg} / \mathrm{mL}$, respectively). All the calculations used in the next sections are expressed in dry weight of the extracts. usando o correspondente solvente de extração (água ou acetona). Após a secagem, as placas de ccd foram pulverizadas com uma solução de DPPH $0,2 \%$ (V/V) em metanol e analisadas 10 min. após a pulverização. $\mathrm{O}$ BHT e a quercetina foram usados como controlos positivos. Os extratos com capacidade de reduzir o radical DPPH desenvolveram manchas amarelas contra um fundo roxo.

\section{Resultados}

Neste trabalho, os extratos das plantas inteiras de Plectranthus hadiensis (variedade tomentosus), Plectranthus madagascarensis, Plectranthus neochilus, Plectranthus verticillatus and Plectranthus barbatus foram preparados por diferentes métodos de extração. De modo a estabelecer os resultados em função do peso de extrato seco (no caso dos extratos aquosos) $1 \mathrm{~mL}$ de cada extrato foi liofilizado e foi determinado o seu peso de extrato seco. Os resultados estão apresentados na Tabela 1. Assim, é possivel verificar que $P$. hadiensis (variedade tomentosus) - decocção e P. barbatus infusão originaram os extratos com os rendimentos mais elevados ( $32 \pm 2,0$ e $33 \pm 3,0 \mathrm{mg} / \mathrm{mL}$, respetivamente). Todos os cálculos obtidos nas secções seguintes estão expressos em termos de extratos seco de planta.

Table 1- Aqueous extracts weight of dry plant extracts $(\mathrm{mg} / \mathrm{mL})$ for each Plectranthus plants and extraction methods.

Tabela 1- Peso dos extractos aquosos das plantas secas $(\mathrm{mg} / \mathrm{mL})$ para cada planta de Plectranthus e métodos de extracção.

\begin{tabular}{|c|c|c|}
\hline Plant / Planta & Extraction method / Método de Extração & $\begin{array}{c}\begin{array}{c}\text { Weight of dry plant extract } \\
(\mathrm{mg} / \mathrm{mL})\end{array} \\
\text { Peso de extrato de planta seca }(\mathrm{mg} / \mathrm{mL})\end{array}$ \\
\hline \multirow{3}{*}{ P. neochilus } & Decoction / Decocção & $22 \pm 2,0$ \\
\hline & Infusion / Infusão & $23 \pm 10,0$ \\
\hline & Microwave / MW (micro-ondas) & $11 \pm 4,0$ \\
\hline \multirow{3}{*}{ P. madagascarensis } & Decoction / Decocção & $22 \pm 1,0$ \\
\hline & Infusion / Infusão & $26 \pm 4,0$ \\
\hline & Microwave / MW (micro-ondas) & $15 \pm 4,0$ \\
\hline \multirow{3}{*}{$\begin{array}{c}\text { P. hadiensis } \\
\text { (var. tomentosus) }\end{array}$} & Decoction / Decocção & $32 \pm 2,0$ \\
\hline & Infusion / Infusão & $26 \pm 1,0$ \\
\hline & Microwave / MW (micro-ondas) & $17 \pm 1,0$ \\
\hline \multirow{3}{*}{ P. barbatus } & Decoction / Decocção & $26 \pm 2,0$ \\
\hline & Infusion / Infusão & $33 \pm 3,0$ \\
\hline & Microwave / MW (micro-ondas) & $22 \pm 1,0$ \\
\hline \multirow{3}{*}{ P. verticillatus } & Decoction / Decocção & N.D \\
\hline & Infusion / Infusão & N.D \\
\hline & Microwave / MW (micro-ondas) & 12 \\
\hline
\end{tabular}


The acetonic extracts yielded residues that are shown in Table 2 where $P$. neochilus presented the highest acetonic extracts.
Os extratos acetónicos originaram resíduos que estão apresentados na Tabela 2 , onde $P$. neochilus foi o planta que obteve maior quantidade de resíduo de extrato.

Table 2 - Yield of acetonic extracts (mg) for each Plectranthus plants and extraction methods.

Tabela 2 - Rendimento dos extratos acetónico (mg) de cada planta de Plectranthus e métodos de extracção.

\begin{tabular}{|c|c|c|}
\hline Plant / Planta & $\begin{array}{l}\text { Extraction method } \\
\text { Tipo de extração }\end{array}$ & $\begin{array}{c}\text { Extract yield (mg) } \\
\text { Rendimento do extracto obtido } \\
\text { (mg) }\end{array}$ \\
\hline \multirow{2}{*}{ P. neochilus } & Ultrasound / Ultrasons & 107,0 \\
\hline & Maceration / Maceração & 50,7 \\
\hline \multirow{2}{*}{ P. madagascarensis } & Ultrasound / Ultrasons & 8,1 \\
\hline & Maceration / Maceração & 41,6 \\
\hline \multirow{2}{*}{$\begin{array}{c}\text { P. hadiensis } \\
\text { (variedade tomentosus) }\end{array}$} & Ultrasound / Ultrasons & 98,5 \\
\hline & Maceration / Maceração & 9,8 \\
\hline \multirow{2}{*}{ P. barbatus } & Ultrasound / Ultrasons & 55,7 \\
\hline & Maceration / Maceração & 41,0 \\
\hline \multirow[t]{2}{*}{ P. verticillatus } & Ultrasound / Ultrasons & ND \\
\hline & Maceration / Maceração & 15,0 \\
\hline
\end{tabular}

\section{Antibacterial activity}

The antibacterial activity of the extracts of five species of the Plectranthus genus on five bacteria was evaluated by the well diffusion assay. Among the studied extracts only the acetonic ones exhibited antibacterial activity (see Table 3 ) and the remaining aqueous extracts were not active against the strains tested. None of the extracts showed activity against Gram-negative bacteria, since their inhibition zones were similar to those of DMSO $(5 \mathrm{~mm})$ used as the negative control (data not shown).

The most pronounced activity, with inhibition zones higher than $20 \mathrm{~mm}$, was shown by the acetonic extract of $P$. madagascarensis against all three Gram-positive bacteria tested. This extract was as active as vancomycin, used as the positive control against the Gram-positive bacteria. $P$. hadiensis $(v d), P$. verticillatus and $P$. madagascarensis extracts presented activity against some or all of the Grampositive bacteria tested. The $P$. hadiensis $(v d)$ extract

\section{Atividade antibacteriana}

A actividade antibacteriana dos extractos de cinco espécies do género Plectranthus foi avaliada em cinco bactérias pelo método da difusão em poço. De todos os extratos estudados só os extratos acetónicos mostraram atividade antibacteriana (Tabela 3), os restantes extratos aquosos não mostraram ser ativos nas bactérias testadas neste estudo. Nenhum dos extractos mostrou actividade antibacteriana contra bactérias Gram negativas, apresentando zonas de inibição iguais à obtida com o controlo negativo DMSO (5 mm; dados não apresentados).

A actividade mais acentuada, com zonas de inibição superiores a $20 \mathrm{~mm}$, foi obtida com o extracto acetónico de $P$. madagascarensis contra as três bactérias Gram-positivas testadas. Este extrato foi tão ativo como a vancomicina, usada como controlo positivo para as bactérias Gram-positivas. Os resultados mostraram que algumas ou todas as bactérias Gram-positivas testadas foram sensíveis aos 
only exhibited activity against $B$. subtilis while the $P$. verticillatus extract showed antimicrobial activity against $S$. aureus and $B$. subtilis (Table 3 ). extratos de $P$. madagascarensis, $P$. verticillatus e $P$. hadiensis. $\mathrm{O}$ extrato de $P$. hadiensis ( $v d$ ) apresentou actividade apenas contra $B$. subtilis enquanto que o extracto de $P$. verticillatus mostrou atividade antimicrobiana contra $S$. aureus e o B. subtilis (ver Tabela 3)

Table 3 - Antibacterial activity of Plectranthus spp.: P. m., P. madagascarensis; P. h., P. hadiensis; P. v., P. verticillatus; $\mathrm{U}$, acetonic extract obtained by ultrasoun; $\mathrm{AM}$, acetonic extract obtained by maceration; -, no activity.

${ }^{\mathrm{b}}$ inhibition zones including the diameter of the diffusion well $(5 \mathrm{~mm})$

${ }^{a}$ vancomycin $(1 \mathrm{mg} / \mathrm{ml})$ for Gram-positive bacteria; norfloxacin $(1 \mathrm{mg} / \mathrm{ml})$ for Gram-negative bacteria

Tabela 3 -Atividade antibacteriana de spp. Plectranthus: P. m., P. madagascarensis; P. h., P. hadiensis; P. v., P. verticillatus; U, extracto acetónico obtido por ultrasons; AM, extracto acetónico obtido por maceração; -, nenhuma actividade

${ }^{\mathrm{b}}$ zona de inibição, incluindo o diâmetro do poço $(5 \mathrm{~mm})$

a vancomicina $(1 \mathrm{mg} / \mathrm{ml})$ para bactérias Gram-positivas; norfloxacina $(1 \mathrm{mg} / \mathrm{ml})$ para bactérias Gramnegativas

\begin{tabular}{lccccc}
\hline \multirow{2}{*}{ Strain / Estirpe } & \multicolumn{4}{l}{ Inhibition Zone $(\mathrm{mm})^{\mathrm{a} /}$ Zona de Inibição $(\mathrm{mm})^{a}$} \\
\cline { 2 - 5 } & $P . m$. (AU) & P. m. (AM) & P. h. (AM) & P. v. (AM) & ${\text { Antibiotic } \text { Antibiotico }^{b}}^{\text {Gram positive / positiva }}$ \\
S. aureus & 26 & 28 & - & 9 & 22 \\
E. faecalis & 21 & 23 & - & - & 17 \\
B. subtilis & 36 & 36 & 7 & 9 & 39
\end{tabular}

Gram negative / negativa

E. coli

P. aeruginosa

\section{Antioxidant activity}

The antioxidant activity of the Plectranthus species aqueous extracts which were obtained by infusion, decoction and microwave, and the acetonic extracts which were obtained by maceration and ultrasounds, was evaluated using the DPPH bleaching method. This method gives information about the capacity of the tested extracts to scavenge free radicals. The results showed that almost all extracts appeared as yellow spots against a purple background, except for both $P$. hadiensis (variety tomentosus), maceration and ultrasound acetonic extracts. These showed purple colored spots and thus no antioxidant activity. The

\section{Actividade antioxidante}

A atividade antioxidante dos extratos aquosos obtidos por infusão, decocção e microondas e dos extratos acetónicos obtidos por maceração e ultrasons das espécies de Plectranthus foi avaliada através do teste de descoloração de DPPH. Este método fornece informação sobre a capacidade dos extratos testados para captar os radicais livres.. Os resultados mostraram que quase todos os extractos apareceram como manchas amarelas contra um fundo roxo, exceto para $P$. hadiensis (variedade tomentosus) para ambos os métodos de extracção com acetona, por maceração e por ultrasons, que apresentaram manchas de cor 
yellow spots of the antioxidant extracts were similar to those of the positive controls, the antioxidant agents $\mathrm{BHT}$ and the quercetin.

\section{Discussion}

In the present study, the screening of antioxidant and antimicrobial activities of five species of Plectranthus genus (P. neochilus, $P$. madagascarensis, $P$. hadiensis (variety tomentosus), $P$. barbatus and $P$. verticillatus) was analyzed. Different extraction methods were studied (decoction, infusion and microwave methods for aqueous extractions, and ultrasounds and maceration for acetonic extractions) with water and acetone as extraction solvent ${ }^{(15)}$. The aim of this study was to search for significant changes in terms of yields and chemical composition. In terms of yields the results did not show typical marked effects (Table 1 and 2) for each extraction method but the two different solvent extracts studied (water and acetone) revealed different biological activities (Table 3). Thus, the antimicrobial and antioxidant acetonic extracts, in contrast to the aqueous extracts, point to different chemical composition. This should be elucidated in future studies.

The in vitro screening for antimicrobial activity was carried out using Gram-positive bacteria (Staphylococcus aureus, Enterococcus faecalis and B. subtilis) and Gram-negative bacteria (E. coli and $P$. aeruginosa). Only the acetonic extracts of $P$. madagascarensis (obtained by maceration and ultrasounds) and $P$. hadiensis and $P$. verticillatus (obtained by maceration) showed positive results against Gram-positive bacteria (Table 3). That is in agreement with other reported studies of Plectranthus genus plants ${ }^{(16)}$ which also do not show activity against Gram negative bacteria. P. madagascariensis was shown to be as active as vancomycin against Grampositive strains and therefore the evaluation of MIC values (minimum inhibitory concentration) should be performed to complete this study. These extracts should be further phytochemically analyzed to identify their bioactive compounds.

The aqueous extracts were inactive against all the bacteria tested but, like the acetonic extracts, they were shown to be antioxidant (except $P$. hadiensis (var. tomentosus)). This result is also in agreement with other studies of Plectranthus extracts ${ }^{(17)}$. The antioxidant activity was qualitatively screened but these antioxidant extracts can be further quantitatively studied for the evaluation of their extract concentration that gave $50 \%$ of antioxidant activity $\left(\mathrm{IC}_{50}\right)$. Other bioactivity studies should be carried out in order to púrpura, e, portanto, desprovidos de actividade antioxidante. As manchas amarelas dos extratos antioxidantes foram semelhantes às manchas dos controlos positivos, os agentes antioxidantes BHT e quercetina.

\section{Discussão}

No presente estudo, foram pesquisadas as atividades antioxidante e antimicrobiana de cinco espécies do género Plectranthus ( $P$. neochilus, $P$. madagascarensis, $P$. hadiensis (variedade tomentosus), P. barbatus e P. verticillatus). Diferentes métodos de extracção foram estudados (para os extratos aquosos foram realizados os métodos de infusão, decocção e microondas e ultra-sons e maceração para os extratos acetónicos), utilizando como solventes de extração a água e a acetona ${ }^{(15)}$. O objetivo deste estudo foi o de pesquisar diferenças significativas em termos de rendimento e composição química nos diversos extractos. Em termos de rendimento, os resultados não mostraram efeitos marcados típicos para cada método de extracção (Tabela 1 e 2), mas os extractos obtidos com os dois solventes estudados (água e acetona) revelaram diferentes actividades biológicas (Tabela 3). Deste modo, as diferenças nas atividades antibacteriana e antioxidante dos extratos acetónicos e dos extratos aquosos indicam, muito provavelmente, uma composição química diferente, o que deve ser esclarecido em estudos posteriores.

$\mathrm{O}$ rastreio in vitro da atividade antimicrobiana foi realizado utilizando bactérias Gram-positivas (Staphylococcus aureus, Enterococcus faecalis e B. subtilis) e bactérias Gram-negativas ( $E$. coli e $P$. aeruginosa). Apenas os extratos acetónico de $P$. madagascarensis (obtidos por maceração e ultra-sons) e $P$. hadiensis e $P$. verticillatus (obtidos por maceração) mostraram resultados positivos contra bactérias Grampositivas (Tabela 3 ). Isso está de acordo com outros estudos descritos para plantas do género Plectranthus ${ }^{(16)}$ que também não revelaram ser ativos em bactérias Gram-negativas. O extrato de $P$. madagascariensis demonstrou ser tão activo como a vancomicina, contra as bactérias Gram-positivas testadas e, portanto, a avaliação dos valores de CMI (concentração mínima inibitória) devem ser realizados para completar este estudo. Estes extratos devem ser alvo de estudos fitoquímicos para a identificação dos seus compostos bioactivos.

Os extratos aquosos mostraram ser inativos contra todas as bactérias testadas, mas estes extratos bem como os extratos acetónicos demostraram ser antioxidantes (excepto P. hadiensis (var. tomentosus)). Este resultado também está de acordo com outros 
unravel other potential bioactivities. The acetonic extracts exhibited antioxidant and antimicrobial activities and thus should be quantitatively evaluated $\left(\mathrm{IC}_{50}\right.$ and MIC respectively). Moreover, future studies must be done to identify which are the bioactive compounds responsible for these bioactivities. $P$. madagascariensis displayed excellent antimicrobial activity and revealed antioxidant activity. Therefore it is a promising starting material for additional studies. Consequently, this bioactivity screening of Plectranthus extracts is important as a preliminary study to select which plants should be investigated further to obtain relevant conclusions about these medicinal plants.

\section{Conclusions}

In the present study, five species of Plectranthus genus were analyzed to evaluate their antimicrobial and antioxidant activities. Different methods were considered for each extraction solvent to search for significant changes in yields but the results showed no marked effects. It was revealed that the two extraction solvents studied, water and acetone, have different biological activities. Considering the antimicrobial activity, only the acetonic extracts showed positive results against Gram-positive bacteria and $P$. madagascariensis was as active as vancomycin against the Gram-positive bacteria. The aqueous extracts were inactive against all the bacteria tested. All aqueous extracts, as well as acetonic extracts (except $P$. hadiensis (var. tomentosus)) showed antioxidant activity. Although aqueous extracts showed no antimicrobial activity, they displayed antioxidant activity and thus further studies should be carried out in order to unravel other potential bioactivities. The acetonic extracts exhibited antioxidant and antimicrobial activities and thus should be evaluated quantitatively. Studies should be undertaken to identify the bioactive compounds responsible for these bioactivities. $P$. madagascariensis displayed excellent antimicrobial activity and demonstrated antioxidant activity. Therefore it is a promising starting material for additional studies. estudos de extratos de Plectranthus ${ }^{(17)}$. A atividade antioxidante foi qualitativamente rastreada mas estes extratos antioxidantes pode ser estudados quantitativamente para a avaliação da concentração de extrato, que origina $50 \%$ da atividade antioxidante $\left(\mathrm{IC}_{50}\right)$. Estudos adicionais poderão também ser realizados com o objectivo de desvendar outras potenciais bioactividades. Os extratos acetónico exibiram atividades antioxidante e antimicrobiana e, portanto, também devem ser avaliados quantitativamente (avaliação dos valores $\mathrm{IC}_{50}$ e CMI, respetivamente). Outros estudos futuros importantes são a elucidação dos compostos bioativos responsáveis pelas actividades demonstradas. $P$. madagascariensis exibiu uma excelente atividade antimicrobiana e revelou atividade antioxidante e por isso é um material de partida promissor para estudos adicionais. Consequentemente, esta triagem de bioatividade de extratos de Plectranthus é importante como um estudo preliminar para selecionar quais plantas que devem ser investigadas para obter conclusões relevantes sobre estas plantas medicinais.

\section{Conclusões}

No presente estudo, cinco espécies do género Plectranthus foram analisadas para testar as suas actividades antimicrobiana e antioxidante. Diferentes métodos de extração foram considerados para cada solvente extractor de modo a pesquisar alterações significativas nos rendimentos, mas os resultados não mostraram diferenças significativas. Os dois solventes de extracção estudados, água e acetona, revelaram diferentes atividades biológicas. Considerando a actividade antimicrobiana, apenas os extratos acetónico mostraram resultados positivos contra bactérias Grampositivas e $P$. madagascariensis mostrou ser tão activo como a vancomicina contra as bactérias Gram-positivas testadas. Os extractos aquosos mostraram ser inactivos contra todas as bactérias testadas, mas estes e os extratos acetónicos demostraram possuir actividade antioxidante (excepto P. hadiensis (var. tomentosus)). Os extractos aquosos não revelaram ter ação antimicrobiana mas demostraram possuir atividade antioxidante e, portanto, novos estudos devem ser realizados a fim de desvendar outras potenciais bioactividades. Uma vez que os extratos acetónicos exibiram atividades antioxidante e antimicrobiana deverão ser posteriormente avaliados quantitativamente e futuros estudos devem ser realizados para identificar os compostos bioativos responsáveis pela atividade demonstrada. $P$. madagascariensis apresentou atividade antimicrobiana excelente e revelou ser antioxidante e por isso é um material de partida promissor para estudos adicionais. 


\section{Acknowledgments}

The authors thank Professor Aida Duarte, Faculty of Pharmacy, University of Lisbon for giving the bacteria tested.

\section{Conflict of Interest}

The authors declare that there is no financial or personal relationship that can be understood as representing a potential conflict of interest.

\section{Agradecimentos}

Os autores agradecem à Professora Aida Duarte da Faculdade de Farmácia da Universidade de Lisboa por ceder as bactérias testadas.

\section{Conflito de Interesses}

Os autores declaram a inexistência de qualquer tipo de relação financeira ou pessoal que possa representar um potencial conflito de interesses.

\section{References / Referências}

[1]. Pan L., Chai H., Kinghorn A.D., The continuing search for antitumor agents from higher plants, Phytochemistry Letters, 2010, 3, 1-8.

[2]. Matu E. N., van Staden. J., Antibacterial and antiinflammatory activities of some plants used for medicinal purposes in Kenya, Journal of Ethnopharmacology, 2003, 87, 35-41.

[3]. P. Rijo, B. Rodríguez, A. Duarte, M. F. Simões "Antimicrobial properties of Plectranthus ornatus extracts, 11-acetoxyhalima-5,13-dien-15-oic acid metabolite and its derivatives", The Natural Products Journal, 2011, 1, 57-64.

[4]. P. Rijo, C. Gaspar-Marques, María F. Simões, María L. Jimeno, B. Rodríguez, "Further diterpenoids from Plectranthus ornatus and P. grandidentatus", Biochemical systematics and ecology, 2007, 35, 215221.

[5].Ozyurt D., Demirata B., Apak R., Determination of total antioxidant capacity by a new spectrophotometric method based on Ce(IV) reducing capacity measurement, Talanta, 2007, 71, 1155-1165.

[6].Muraina I.A. et al., 2009 Muraina I.A., Suleiman M.M., Eloff J.N., Can MTT be used to quantify the antioxidant activity of plant extracts?, Phytomedicine, 2009, 16, 665-668.

[7].Maioli M.A. et al., 2010 Maioli M. A., Alves L. C., Campanini A. L., Lima M. C., Dorta D. J., Groppo M., Cavalheiro A. J., Curti C., Mingatto F. E., Iron chelating- mediated antioxidant activity of Plectranthus barbatus extract on mitochondria, Food Chemistry, 2010, 122 203-208.

Raja S., Naresh R., Kumar

[8]. B. S., Evaluation of nephroprotective, diuretic, and antioxidant activities of Plectranthus amboinicus on acetaminophen-induced nephrotoxic rats, Toxicology Mechanisms and Methods, 2010, 20, 4, 213-221.

[9]. Gibbons S., Review: Anti-staphylococcal plant natural products, Nat. Prod. Rep., 2004, 21, 263-277.

[10].Maistry K., Maistry K., The antimicrobial properties and chemical composition of leaf essential oils of indigenous Plectranthus (Lamiaceae) species, $\mathrm{PhD}$ thesis of University of the Witwatersrand, Johannesburg, South Africa, 2003

[11]. Rijo P., Phytochemical study and biological activities of diterpenes and derivatives from Plectranthus species, $\mathrm{PhD}$ thesis of University of Lisbon, Lisbon, Portugal, 2011

[12]. CLSI- Clinical and Laboratory Standards Institute (2011). Performance standards for antimicrobial susceptibility testing: Twenty First International Supplement M100-S21. Clinical and Laboratory Standards Institute, Wayne, PA.

[13]. Pisoschi A.M., Cheregi M. C., Danet A. F., Total Antioxidant Capacity of Some Commercial Fruit Juices: Electrochemical and Spectrophotometrical Approaches, Molecules, 2009, 14, 480-493.
[14].Sofidiya M.O., Odukoya O.A., Afolayan A.J., Familoni O.B., Phenolic contents, antioxidant and antibacterial activities of Hymenocardia Acida, Natural Product Research, 2009, 23, 2, 168-177.

[15]. Xi Jun, Shen Deji, Li Ye, Zhang Rui, Comparison of in vitro antioxidant activities and bioactive components of green tea extracts by different extraction methods, International Journal of Pharmaceutics, 2011, 408 97-101.

[16].C. Gaspar-Marques, Patrícia Rijo, M. Fátima Simões, M. Aida Duarte e Benjamin Rodriguez, "Abietanes from Plectranthus grandidentatus and P. hereroensis against methicillin- and vancomycinresistant bacteria", Phytomedicine, 2006, 13, 267-271.

[17]. Pedro L. Falé, Carlos Borges, Paulo J. Amorim Madeira, Lia Ascensão, M. Eduarda M. Araújo, M. Helena Florêncio, M. Luísa M. Serralheiro, Rosmarinic acid, scutellarein 40-methyl ether 7-O-glucuronide and (16S)-coleon $\mathrm{E}$ are the main compounds responsible for the antiacetylcholinesterase and antioxidant activity in herbal tea of Plectranthus barbatus ("falso boldo"), Food Chemistry, 2009, 114 798-805. 